\title{
Collagen fiber membrane-derived chemically and mechanically durable superhydrophobic membrane for high- performance emulsion separation
}

\author{
Huifang Li, Wan Zheng, Hanzhong Xiao, Baicun Hao, Yujia Wang, Xin Huang ${ }^{*}$ and Bi Shi
}

\begin{abstract}
Developing high-performance separation membrane with good durability is a highly desired while challenging issue. Herein, we reported the successful fabrication of chemically and mechanically durable superhydrophobic membrane that was prepared by embedding UiO-66 as size-sieving sites within the supramolecular fiber structure of collagen fiber membrane (CFM), followed by the polydimethylsiloxane (PDMS) coating. The as-prepared CFM/ UiO-66(12)/PDMS membrane featured capillary effect-enhanced separation flux and homogeneous porous channels guaranteed high separation efficiency. When utilized as double-layer separation membranes, this new type of composite membranes separated various surfactant stabilized water-in-oil microemulsions and nanoemulsions, with the separation efficiency high up to $99.993 \%$ and the flux as high as $973.3 \mathrm{~L} \mathrm{~m}^{-2} \mathrm{~h}^{-1}$. Compared with commercial polytetrafluoro ethylene (PTFE) membrane, the advantage of the double-layer CFM/UiO-66(12)/PDMS membranes in separation flux was evident, which exhibited one order of magnitude higher than that of commercial PTFE membrane. The CFM/UiO-66(12)/PDMS membrane was acid-alkali tolerant, UV-aging resistant and reusable for emulsion separation. Notably, the CFM/UiO-66(12)/PDMS membrane was mechanically durable against strong mechanical abrasion, which was still capable of separating diverse water-in-oil emulsions after the abrasion with sandpaper and assembled as double-layer separation membranes. We anticipate that the combination of CFM and metal organic frameworks (MOFs) is an effective strategy for fabricating high-performance separation membrane with high mechanical and chemical durability.
\end{abstract}

Keywords: collagen fiber membrane, metal organic frameworks, superhydrophobic composite membrane, emulsion separation, mechanical and chemical durability

\section{Introduction}

Emulsification technology has been widely utilized in a variety of industrial processes [1-4], which inevitably generates a large quantity of surfactant-containing emulsion wastewater. The emulsion wastewater has to be treated before discharged. Among various emulsion separation approaches, membrane separation has attracted great interests due to its high separation efficiency and

\footnotetext{
* Correspondence: xhuangscu@163.com; shibi@scu.edu.cn National Engineering Research Center of Clean Technology in Leather Industry, Sichuan University, 610065 Chengdu, P.R. China
}

simple separation process [5-8]. Membrane separation based on size-sieving strategy can be described by the Hagen-Poiseuille equation: $J=\varepsilon \pi r_{p}{ }^{2} \Delta p /(8 \mu L)$, where the flux $(\mathrm{J})$ is proportional to the square of radius $\left(\mathrm{r}_{\mathrm{p}}{ }^{2}\right)$ and inversely proportional to the thickness of the membrane (L) [9]. Normally, there is a trade-off effect for sizesieving membrane since the permeation flux is constrained by the small membrane pore size that is a necessity for separating emulsions with extremely small droplets. To address this issue, current strategy to improve the permeation flux heavily relies on decreasing
Springer Open (c) The Author(s). 2021 Open Access This article is licensed under a Creative Commons Attribution 4.0 International License, which permits use, sharing, adaptation, distribution and reproduction in any medium or format, as long as you give appropriate credit to the original author(s) and the source, provide a link to the Creative Commons licence, and indicate if changes were made. The images or other third party material in this article are included in the article's Creative Commons licence, unless indicated otherwise in a credit line to the material. If material is not included in the article's Creative Commons licence and your intended use is not permitted by statutory regulation or exceeds the permitted use, you will need to obtain permission directly from the copyright holder. To view a copy of this licence, visit http://creativecommons.org/licenses/by/4.0/. 
the membrane thickness as much as possible [10]. Unfortunately, ultrathin membranes usually suffer from weak mechanical strength, which compromise their application [11]. Therefore, it is highly desirable to develop new separation membranes that are capable of exhibiting high separation performances and still providing good mechanical strength.

Leather, prepared from the tanning of skins/hides, has been utilized as the raw material for shoes, clothes and gloves for thousands of years due to its outstanding mechanical strength $[12,13]$. The tensile strength of leather reaches up to $38 \mathrm{MPa}$ [14], and the tear strength attains $78 \mathrm{~N} \mathrm{~mm}^{-1}$ [15]. Leather is formed by supramolecular self-assembly of type I collagen molecules, which actually can be considered as a type of mechanical durable natural membrane (i.e. collagen fiber membrane, CFM) that features a hierarchically fibrous structure from nanoscale to microscale and macroscale. Our previous investigations have verified that the supramolecular fiber structure of type I collagen molecules exhibited the merits of capillary effect enhanced liquid transport kinetics [16]. In consideration of that type I collagen molecules are the component of CFM, CFM is therefore a promising substrate for fabricating mechanical durable and high-performance separation membranes. However, the poly-dispersed pore size of CFM could fail to separate emulsions with small droplet size because the voids among the fibers of CFM can be considerably larger than the particle size of emulsion droplets to be separated, which are tend to directly penetrate the CFM without demulsification. Metal organic frameworks (MOFs), composed of an inorganic metal center and organic linkers [17], have showed great potential in diverse applications, such as gas separation, gas storage and catalysis [18-20]. Besides large specific surface area and high porosity, the homogeneous porous structure with controllable pore-size makes MOFs as a promising demulsifying component for both microemulsions and nanoemulsions.

Herein, we synthesized a new type of superhydrophobic composite membrane by embedding MOFs as sizesieving sites within the three dimensional (3D) supramolecular fiber structure of CFM, followed by polydimethylsiloxane (PDMS) coating. Specifically, the UiO-66 grown on CFM by seeding growth method was employed as the size-sieving sites since the $\mathrm{UiO}-66$ has high chemical stability and the micropore size of UiO-66 is $\sim 6.0 \AA$, which is capable of demulsifying both microemulsions and nanoemulsions efficiently [21, 22]. For the CFM embedded with UiO-66 (denoted as CFM/UiO66), a subsequent PDMS coating was applied to reduce the surface energy and to enhance chemical and mechanical strength, thus obtaining superhydrophobicity and strengthened chemical and mechanical durability. We prepared a series of the CFM/UiO-66(x)/PDMS membranes, where $x(\mathrm{~h})$ is the time of seeding growth of UiO-66. The as-prepared superhydrophobic CFM/UiO66(12)/PDMS membrane exhibited the water contact angle of $158.7^{\circ}$. The double-layer CFM/UiO-66(12)/ PDMS membranes demonstrated a remarkable capacity to separate a variety of surfactants stabilized water-in-oil microemulsions and nanoemulsions with efficiencies higher than $99.99 \%$, including Span 80/SDBS stabilized water-in-dodecane nanoemulsion (NE1), Span 80/CTAB stabilized water-in-dodecane nanoemulsion (NE2), Tween 80/SDBS stabilized water-in-n-octane nanoemulsion (NE3), Tween 80/SDS stabilized water-in-n-heptane nanoemulsion (NE4), Span 80/SDS stabilized water-indodecane microemulsion (ME1), and Span 80/CTAB stabilized water-in-dodecane microemulsion (ME2). The separation flux of the double-layer CFM/UiO-66(12)/ PDMS membranes was superior to that of commercial polytetrafluoro ethylene (PTFE) membrane. When applied for the separation of NE4, the separation flux of the double-layer CFM/UiO-66(12)/PDMS membranes was as high as $973.3 \mathrm{~L} \mathrm{~m}^{-2} \mathrm{~h}^{-1}$, which was 3.18 -fold to the flux of commercial PTFE membrane (305.6 L $\mathrm{m}^{-2} \mathrm{~h}^{-1}$ ). For the separation of NE1, the flux of the double-layer CFM/UiO-66(12)/PDMS membranes (540.4 $\mathrm{L} \mathrm{m}^{-2} \mathrm{~h}^{-1}$ ) was one magnitude higher than that of commercial PTFE membrane $\left(77.6 \mathrm{~L} \mathrm{~m}^{-2} \mathrm{~h}^{-1}\right)$. Moreover, the double-layer CFM/UiO-66(12)/PDMS membranes were able to be reused for emulsion separation, with separation efficiency high up to $99.993 \%$ in the 5th separation cycle of NE2. The CFM/UiO-66(12)/ PDMS membranes remained superhydrophobic after subjected to harsh environments, such as organic solvent immersion, ultraviolet irradiation, strong acid immersion and strong alkali immersion. Notably, the double-layer CFM/UiO-66(12)/PDMS membranes assembled by two CFM/UiO-66(12)/PDMS membranes that were treated by abrasion with sandpaper for 200 cycles still exhibited high-performance separation capability in separating above 6 type of microemulsions and nanoemulsions, with the separation flux as high as $1012.2 \mathrm{~L} \mathrm{~m}^{-2} \mathrm{~h}^{-1}$ and the efficiency high up to $99.993 \%$. Therefore, we provided a new strategy for developing chemically and mechanically durable membrane that was highly efficient for highperformance separation of water-in-oil emulsions.

\section{Experimental section}

\subsection{Materials}

Chrome tanned split-leather was supplied by Ruixing Leather Co., Ltd (Haining, China). $\mathrm{ZrCl}_{4}$ ( $\geq 99.9 \%$ ), zirconium (IV) propoxide solution (70wt \%) and 1,4-benzenedicarboxylic acid $\left(\mathrm{H}_{2} \mathrm{BDC}\right)$ were purchased from Aladdin Reagent Co. (Shanghai, China). Polydimethylsiloxane (PDMS) prepolymer (Sylgard 184A) and curing 
agent (Sylgard 184B) were purchased from Dow Corning Corporation (Midland, USA). The PDMS solution was prepared by dissolving $10 \mathrm{~g}$ of Sylgard 184A in $200 \mathrm{~g}$ dodecane and $1.0 \mathrm{~g}$ of Sylgard $184 \mathrm{~B}$ in $20 \mathrm{~g}$ dodecane, followed by mixing above solutions together. Sodium dodecyl benzene sulfonate (SDBS), sodium dodecyl sulfate (SDS), hexadecyl trimethyl ammonium bromide (CTAB), and other chemicals were analytical grade reagents and were used as received without further purification.

\subsection{Synthesis of UiO-66 nanoparticles}

UiO-66 nanoparticles (UiO-66NPs) were prepared according to the literature[23]. $\mathrm{ZrCl}_{4}(0.318 \mathrm{~g})$ was ultrasonically dissolved in $108 \mathrm{~mL}$ of $\mathrm{N}, \mathrm{N}$-Dimethylformamide (DMF), followed by the addition of $\mathrm{H}_{2} \mathrm{BDC}(0.228 \mathrm{~g})$. The mixture was transferred into an autoclave, heated at $120{ }^{\circ} \mathrm{C}$ for $24 \mathrm{~h}$, and then cooled to room temperature. The UiO66NPs were obtained after centrifuged at $8000 \mathrm{rpm}$, and dried under vacuum at $60^{\circ} \mathrm{C}$.

\subsection{Fabrication of the CFM/UiO-66(x)/PDMS membrane}

The collagen fiber membrane (CFM), with the diameter of $8.0 \mathrm{~cm}$ and the thickness of 1.5-1.8 $\mathrm{mm}$ was immersed in $100 \mathrm{~mL}$ of aqueous suspension containing $20 \mathrm{mg}$ of above synthesized UiO-66NPs under ultrasonication for $1.0 \mathrm{~h}$ to obtain the seeded CFM. Then, the seeded CFM was dried at $60{ }^{\circ} \mathrm{C}$. DMF $(70 \mathrm{~mL})$, zirconium (IV) propoxide solution $(0.71 \mathrm{~mL})$ and glacial acetic acid $(40 \mathrm{~mL})$ were mixed, followed by the addition of $\mathrm{H}_{2} \mathrm{BDC}(750 \mathrm{mg})$. Then, the seeded CFM was immersed in above mixture, keeping still for the seeding growth of UiO-66. The resultant products were CFM/ UiO-66 $(x)$, where $x(\mathrm{~h})$ is the time of seeding growth of UiO-66, equaling to 3.0,6.0,9.0,12, 15 and 18. The dried CFM/UiO-66 $(x)$ membranes were immersed in 30 $\mathrm{mL}$ of $5.0 \mathrm{wt} \%$ PDMS solution for $5.0 \mathrm{~min}$. After dried at $70{ }^{\circ} \mathrm{C}$, the CFM/UiO-66(x)/PDMS membranes were obtained.

\subsection{Fabrication of the CFM/PDMS membrane}

The CFM with the diameter of $8.0 \mathrm{~cm}$ and the thickness of $1.5-1.8 \mathrm{~mm}$ was immersed in $30 \mathrm{~mL}$ of $5.0 \mathrm{wt} \%$ PDMS solution for $5.0 \mathrm{~min}$. After dried at $70{ }^{\circ} \mathrm{C}$, the CFM/ PDMS membrane was obtained.

\subsection{Preparation of water-in-oil emulsion}

Various emulsions with droplet sizes from micrometer to nanometer range were prepared. Table S1 summarizes the ingredients of the prepared six types of emulsions, including Span 80/SDBS stabilized water-indodecane nanoemulsion (denoted as NE1), Span 80/ CTAB stabilized water-in-dodecane nanoemulsion (denoted as NE2), Tween 80/SDBS stabilized water-in-n-octane nanoemulsion (denoted as NE3), and Tween 80/
SDS stabilized water-in-n-heptane nanoemulsion (denoted as NE4), Span 80/SDS stabilized water-indodecane microemulsion (denoted as ME1), and Span 80/CTAB stabilized water-in-dodecane microemulsion (denoted as ME2). For the preparation of NE1, Span 80 $(0.12 \mathrm{~g})$ and SDBS $(0.06 \mathrm{~g})$ were dissolved in dodecane $(300 \mathrm{~mL})$ and water $(3.0 \mathrm{~mL})$, respectively. The SDBS water solution was drop-wise added into the Span 80 dodecane solution under agitation at $1000 \mathrm{rpm}$. Then, the resultant mixture was stirred at $2000 \mathrm{rpm}$ for $1.0 \mathrm{~h}$ to obtain NE1. Other emulsions were prepared by the similar method used for preparing NE1.

\subsection{Emulsion separation of the CFM/UiO-66(x)/PDMS membrane}

Emulsion separation was conducted in an H-type equipment (as shown in Figure S1), with the CFM/UiO-66 $(x)$ / PDMS membranes fixed between the feed cell and the collecting cell. The prepared emulsion was poured into the feed cell and the corresponding filtrate was obtained in the collecting cell. The water content in the collected filtrates was measured by Karl Fischer moisture titrator (Wo Ye technology, S-300, China). The separation efficiency was calculated by the following equation: Efficiency $=100 \%$ - $C$, where $C$ is the water content in the filtrate. The flux is calculated as below: $J=\mathrm{V} /(\mathrm{St})$, where $\mathrm{J}$ is the flux of the membrane, $\mathrm{V}$ is the filtrating volume, $\mathrm{S}$ is the area of the membrane, and $\mathrm{t}$ is the time used for emulsion separation.

The reusability of the double-layer CFM/UiO-66(12)/ PDMS membranes was evaluated by repeated separation of NE2 for five cycles. Each separation cycle of NE2 was carried out for $1.0 \mathrm{~h}$, with the flux and water content in the filtrates calculated at the interval of $20 \mathrm{~min}$. After each cycle, the used membranes were washed with ethanol, dried and then applied in the next cycle.

\subsection{Chemical and mechanical durability}

The solvent resistance of the CFM/UiO-66(12)/PDMS membranes was tested by direct immersion in different solvents. Specifically, the CFM/UiO-66(12)/PDMS membranes were immersed in dodecane, $\mathrm{n}$-heptane or $\mathrm{n}$ octane for $24 \mathrm{~h}$. Then, the treated CFM/UiO-66(12)/ PDMS membranes were dried at $70{ }^{\circ} \mathrm{C}$ and their water contact angles (WCAs) were measured.

The acid-alkali resistance was also investigated by immersing the CFM/UiO-66(12)/PDMS membranes in the aqueous solution with the $\mathrm{pH}$ ranging from 2 to 12 . Then, the CFM/UiO-66(12)/PDMS membranes were dried at $70{ }^{\circ} \mathrm{C}$ and their WCAs were measured.

The UV resistance of the CFM/UiO-66(12)/PDMS membrane was tested by exposing it to ultraviolet irradiation for $24 \mathrm{~h}$, followed by WCA test. The power of the UV lamp was $18 \mathrm{~W}$, and the maximum wavelength was $365 \mathrm{~nm}$. 
The mechanical durability of the CFM/UiO-66(12)/ PDMS membrane was examined by abrasion test. The abrasion test was performed on a rub fastness tester (Figure S2). The CFM/UiO-66(12)/PDMS membrane was fixed on the sample table, and a sandpaper (36 mesh, $1.5 \mathrm{~cm} \times 1.5 \mathrm{~cm}$ ) was stuck on the fixed arm. One cycle of abrasion was defined as a forth-and-back friction of the CFM/UiO-66(12)/PDMS membrane against the sandpaper with a total friction distance of $12 \mathrm{~cm}$. The WCAs of the CFM/UiO-66(12)/PDMS membranes abraded by $50,100,150$ and 200 abrasion cycles were measured. The double-layer CFM/UiO-66(12)/PDMS membranes assembled by two membranes that were treated by abrasion with sandpaper for 200 cycles were used for separating a series of water-in-oil microemulsions and nanoemulsions, including NE1, NE2, NE3, NE4, ME1 and ME2.

\subsection{Characterization}

The crystalline structures of UiO-66 and membranes were confirmed by the X-ray diffraction (XRD, Bruker
D8 ADVANCE A25X) with $\mathrm{Cu} K \alpha$ radiation. The surface morphologies of UiO-66 and membranes were observed by a field emission scanning electron microscopy (FESEM, Nova Nanosem 450, America) operated at $3.0 \mathrm{kV}$. Dynamic light scattering (DLS) was measured by the particle size analyzer (Brook haven, NanoBrook, America) for nanoemulsions and the stereomicroscope (Leica, M205 C, Germany) for microemulsions, respectively. Water contents in the filtrates were measured by a Karl Fischer moisture titrator (Wo Ye technology, S-300, China). The wettability of membranes was investigated by water contact angle (Krüss, DSA30, Germany).

\section{Results and discussion}

The schematic illustration in Fig. 1a shows the preparation of the CFM/UiO-66(12)/PDMS membrane (see the preparation details in the Experimental Section). The CFM was simply immersed in aqueous suspension containing UiO-66 nanoparticles (UiO-66NPs) under ultrasonication. In this way, the UiO-66NPs were embedded into the 3D fibrous network of CFM, which served as

(a)
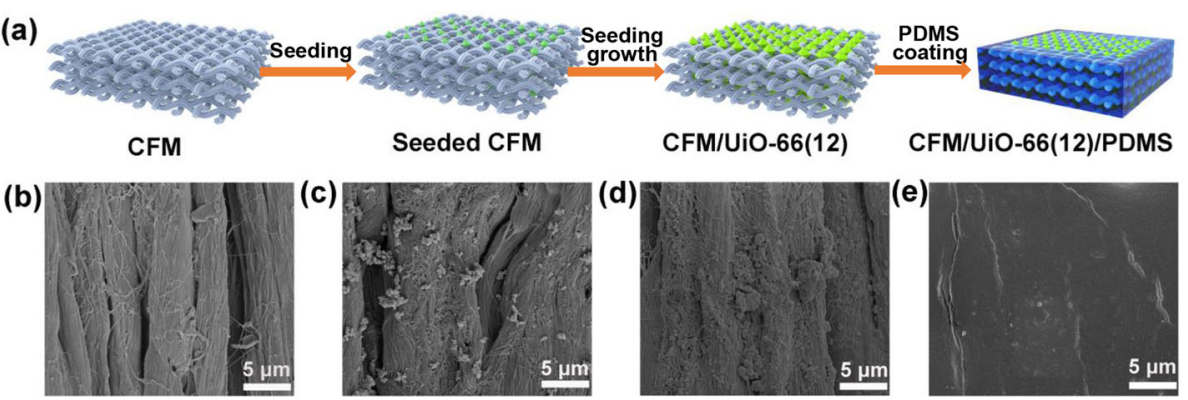

(f)

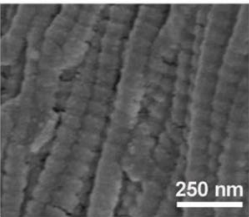

(g)
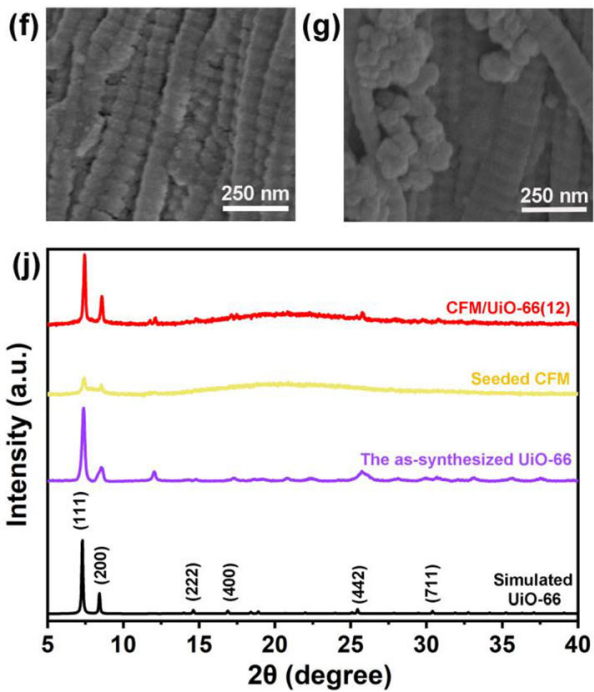

(h)
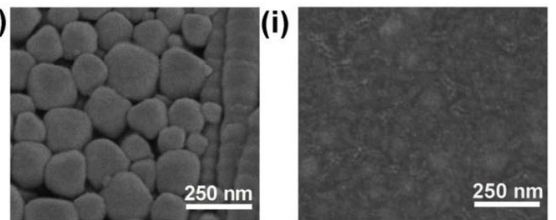

(k)

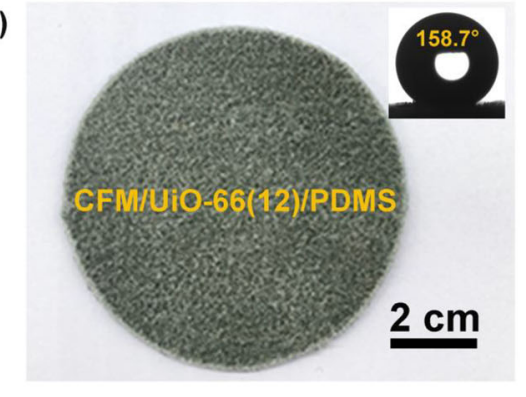

Fig. 1 Schematic illustration showing the fabrication of the CFM/UiO-66(12)/PDMS membrane (a), FESEM images of CFM (b), the seeded CFM (c), the CFM/UiO-66(12) membrane (d) and the CFM/UiO-66(12)/PDMS membrane (e), the higher magnification images of CFM (f), the seeded CFM (g), the CFM/UiO-66(12) membrane (h) and the CFM/UiO-66(12)/PDMS membrane (i), XRD patterns of simulated UiO-66, the as-synthesized UiO66, the seeded CFM and the CFM/UiO-66(12) (j) and the digital photograph of the CFM/UiO-66(12)/PDMS membrane (inset: the corresponding WCA) (k) 
seeds for the further growth of UiO-66 size-sieving sites. As shown in Fig. 1b, f, the CFM has a full fibrous 3D supramolecular fiber structure that has the basic building blocks of collagen nanofiber $(50-200 \mathrm{~nm})$ with the typical $67 \mathrm{~nm}$ D-periodicity. As for the seeded CFM, the UiO-66NPs with the average diameter of $70 \mathrm{~nm}$ have been successfully embedded onto the surface of the CFM (Fig. 1c, g). The seeded CFM was further immersed into $\mathrm{H}_{2} \mathrm{BDC}$-contained zirconium (IV) propoxide solution for the seeding growth of UiO-66NPs. As shown in Fig. 1d, h, the UiO-66NPs with larger particle size $(120 \mathrm{~nm})$ and higher density have been grown on the seeded CFM. These grown UiO-66NPs not only served as the efficient size-sieving sites for emulsion separation but also enhanced the roughness of CFM, which allowed for obtaining superhydrophobicity after a subsequent PDMS coating was applied. After the seeding growth of UiO-66NPs, the resultant CFM/UiO-66(12) membrane was coated with PDMS to reduce the surface energy. As shown in Fig. 1e, i, the UiO-66NPs shows no obvious appearance after the PDMS coating.

The successful preparation of the CFM/UiO-66(12) membrane was also confirmed by XRD patterns (Fig. 1j). The XRD pattern of UiO-66NPs synthesized and used as seeds shows high consistence with that of simulated UiO-66 [23]. As for the seeded CFM, the corresponding XRD pattern shows the crystal planes of (111) and (200) of UiO-66. After the seeding growth, the obtained CFM/ UiO-66(12) membrane shows the diffraction peaks of (111), (200), (222), (400), (442), and (711) crystal planes of the UiO-66. Moreover, the peak intensity of (111) and (200) crystal planes significantly increases as compared with that of the seeded CFM. Above XRD results indicate the successful seeding and seeding growth of $\mathrm{UiO}$ 66NPs on the CFM. For the CFM/UiO-66(12)/PDMS membrane, the mapping images of $\mathrm{O}, \mathrm{Zr}$ and $\mathrm{Si}$ elements confirm the successful growth of UiO-66NPs and the coverage with PDMS layer (Figure S3). The CFM/ UiO-66(12)/PDMS membrane exhibits excellent hydrophobicity, with a water contact angle (WCA) of $158.7^{\circ}$ (Fig. 1k), which is essential for the separation of waterin-oil emulsions.

We prepared a series of the CFM/UiO-66(x)/PDMS membranes, where $x$ was the seeding growth time (h) of UiO-66NPs, varied from 3.0 to 18 . The as-prepared CFM/UiO-66 $(x) /$ PDMS membranes were further applied in the separation of Span 80/SDBS stabilized water-indodecane nanoemulsion (NE1), and we also fabricated the CFM/PDMS membrane without embedding UiO66NPs as the size-sieving sites. The single-layer CFM/ UiO-66( $x) /$ PDMS membranes were firstly utilized in the separation of NE1. As shown in Figure S4, the filtrate collected through the CFM/PDMS membrane is evidently turbid, which suggests that the CFM/PDMS membrane is incapable of separating NE1 without the presence of UiO-66NPs. As the CFM is modified by UiO-66NPs with the seeding growth time increased from 3.0 to $9.0 \mathrm{~h}$, the dynamic light scattering (DLS) curves of the corresponding filtrates exhibit an obvious shift of the particle size range, from 991-1369 nm to 305-459 nm (Figure S5). Evidently, the seeding growth of UiO-66NPs on the seed CFM is a time-dependent process, which enhances the separation capability of the CFM/UiO-66(x)/PDMS membrane with a longer growth time. Figure $\mathrm{S} 6$ shows the difference in the turbidity of the filtrates obtained by the CFM/UiO-66(x)/PDMS membranes. When the seeding growth time is shorter than $12 \mathrm{~h}$, the filtrates separated by the CFM/UiO-66 $(x) /$ PDMS membranes are cloudy. The filtrates are almost transparent when the CFM/UiO-66(x)/PDMS membranes $(x=12,15,18)$ are used for NE1 separation, which is consistent with their increased emulsion separation capability.

Since the single-layer CFM/UiO-66(x)/PDMS membranes were unable to separate NE1 completely, we applied the double-layer CFM/UiO-66(x)/PDMS membranes for separating NE1. As the seeding growth time of UiO-66NPs increases from 3.0 to $9.0 \mathrm{~h}$, the particle size distributions of the collected filtrates separated by the double-layer CFM/ $\mathrm{UiO}-66(x) / \mathrm{PDMS}$ membranes shift from $452-697 \mathrm{~nm}$ to the smaller particle size distribution of 95-129 nm (Fig. 2bd). In the separation of NE1 with the double-layer CFM/ UiO-66(12)/PDMS membranes, no particle size distribution is detected in the DLS curve of collected filtrate (Fig. 2e). The corresponding water content is $81.6 \mathrm{ppm}$ and the separation efficiency is as high as $99.992 \%$ (Fig. 2f). These results indicate that the NE1 has been successfully separated by the double-layer CFM/UiO-66(12)/PDMS membranes. The double-layer CFM/UiO-66(15)/PDMS membranes and the CFM/UiO-66(18)/PDMS membranes are also capable of separating NE1, with the separation efficiencies higher than 99.99 \% (Fig. 2f). However, the separation fluxes of the double-layer CFM/UiO-66(15)/PDMS membranes (510.3 L $\mathrm{m}^{-2} \mathrm{~h}^{-1}$ ) and the CFM/UiO-66(18)/PDMS membranes $\left(502.6 \mathrm{~L} \mathrm{~m}^{-2} \mathrm{~h}^{-1}\right)$ are lower than that of the double-layer CFM/UiO-66(12)/PDMS membranes (540.4 $\mathrm{L} \mathrm{m}^{-2} \mathrm{~h}^{-1}$ ) (Fig. 2f). As a result, the double-layer CFM/UiO-66(12)/ PDMS membranes were employed for the separation of the following microemulsions and nanoemulsions.

We envisaged that the difference in separation performances among the double-layer CFM/UiO-66 $(x) / \mathrm{PDMS}$ membranes should be attributed to the varied amount of $\mathrm{UiO}-66 \mathrm{NPs}$ embedded on the CFM. We compared the field emission scanning electron microscopy (FESEM) images of the CFM/UiO-66(3), CFM/UiO-66(12) and CFM/UiO-66(18) membranes. As shown in Figure S7a, when the seeding growth time of UiO-66NPs is $3.0 \mathrm{~h}$, relatively fewer UiO-66NPs are embedded on the surface 

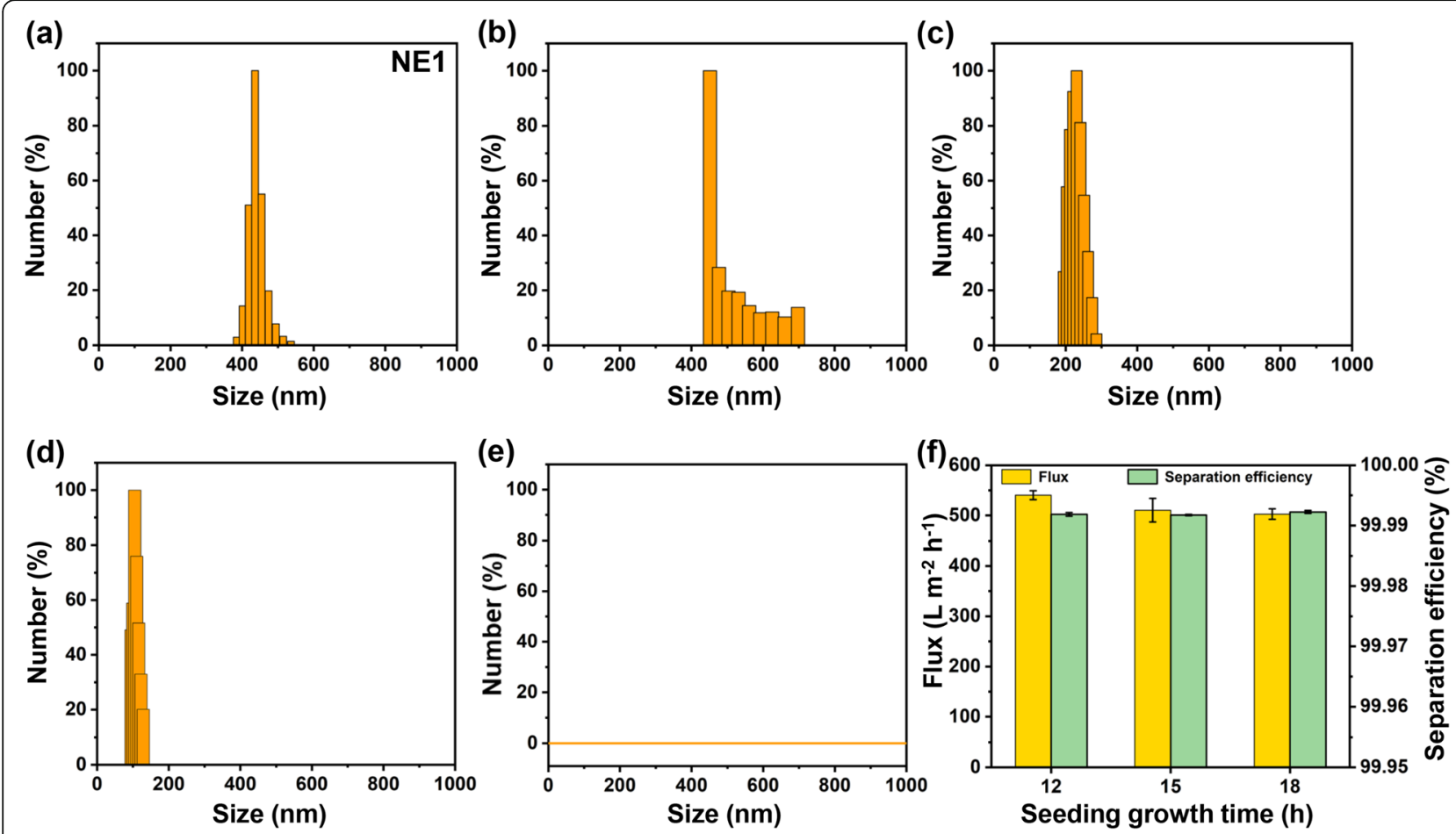

Fig. 2 DLS curves of NE1 before (a) and after the separation by the double-layer CFM/UiO-66(x)/PDMS membranes that were prepared by

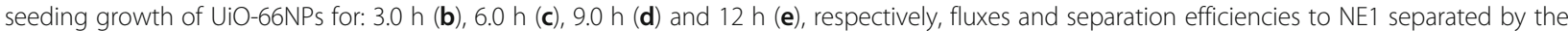
double-layer CFM/UiO-66(x)/PDMS membranes with different seeding growth time of UiO-66NPs (f)

of the CFM/UiO-66(3). Due to the inefficient sizesieving sites of $\mathrm{UiO}-66 \mathrm{NPs}$, the emulsion droplets can directly penetrate through the double-layer $\mathrm{CFM} / \mathrm{UiO}$ 66(3)/PDMS membranes, thus failing to separate the emulsion. As the seeding growth time of UiO-66NPs increases to $12 \mathrm{~h}$, the UiO-66NPs grown on the CFM become larger and denser (Fig. 1d). Accordingly, the separation capability of the double-layer CFM/UiO66(12)/PDMS membranes is enhanced sufficiently to separate the emulsion. As the UiO-66NPs continuously grow for $18 \mathrm{~h}$ with larger particle size and denser packing, the pathway through the micro channels of UiO66NPs will enlarge mass transfer resistance and decrease the separation flux (Figure S7b). We proposed a sizesieving mechanism for the separation of water-in-oil emulsions by the double-layer CFM/UiO-66(12)/PDMS membranes. The micropore size of UiO-66 is $\sim 6.0 \AA$, which is smaller than the particle size of emulsion droplets, thus facilitating the demulsification of water-in-oil emulsion droplets. After the demulsification, the permeation of water phase is strictly prevented by the superhydrophobic double-layer CFM/UiO-66(12)/PDMS membranes while the selective permeation of oil phase through the superhydrophobic double-layer CFM/UiO-66(12)/PDMS membranes is allowed.

A variety of mixed surfactant-stabilized water-in-oil microemulsions and nanoemulsions. were separated by the double-layer CFM/UiO-66(12)/PDMS membranes to evaluate their separation performances to other waterin-oil emulsions. As shown in Fig. 3a-c, Figure S8, the double-layer CFM/UiO-66(12)/PDMS membranes are capable of efficiently separating diverse nanoemulsions, including Span 80/CTAB stabilized water-in-dodecane nanoemulsion (NE2), Tween 80/SDBS stabilized waterin-n-octane nanoemulsion (NE3) and Tween 80/SDS stabilized water-in-n-heptane nanoemulsion (NE4). The double-layer CFM/UiO-66(12)/PDMS membranes are also able to separate microemulsions. As shown in Fig. 3d, the droplets of Span 80/SDS stabilized microemulsion (ME1) can be clearly observed under the optical microscopy. In contrast, no emulsion droplets are found in the filtrate collected through the double-layer CFM/ UiO-66(12)/PDMS membranes (Fig. 3f). As shown in Figure S9, the separation of double-layer CFM/UiO66(12)/PDMS membranes to Span 80/CTAB stabilized water-in-dodecane microemulsion (ME2) is also achieved. The separation fluxes of NE2, NE3, NE4, ME1 and ME2 separated by the double-layer CFM/UiO66(12)/PDMS membranes are $504.6 \mathrm{~L} \mathrm{~m}^{-2} \mathrm{~h}^{-1}, 969.8 \mathrm{~L}$ $\mathrm{m}^{-2} \mathrm{~h}^{-1}, 973.3 \mathrm{~L} \mathrm{~m}^{-2} \mathrm{~h}^{-1}, 545.4 \mathrm{~L} \mathrm{~m}^{-2} \mathrm{~h}^{-1}$ and $351.1 \mathrm{~L}$ $\mathrm{m}^{-2} \mathrm{~h}^{-1}$, respectively (Fig. 3g). The water contents in the filtrates of NE2, NE3, NE4, ME1 and ME2 separated by the CFM/UiO-66(12)/PDMS are $85.1 \mathrm{ppm}, 83.1 \mathrm{ppm}$, $84.8 \mathrm{ppm}, 84.1 \mathrm{ppm}$ and $69.4 \mathrm{ppm}$, respectively, with the 


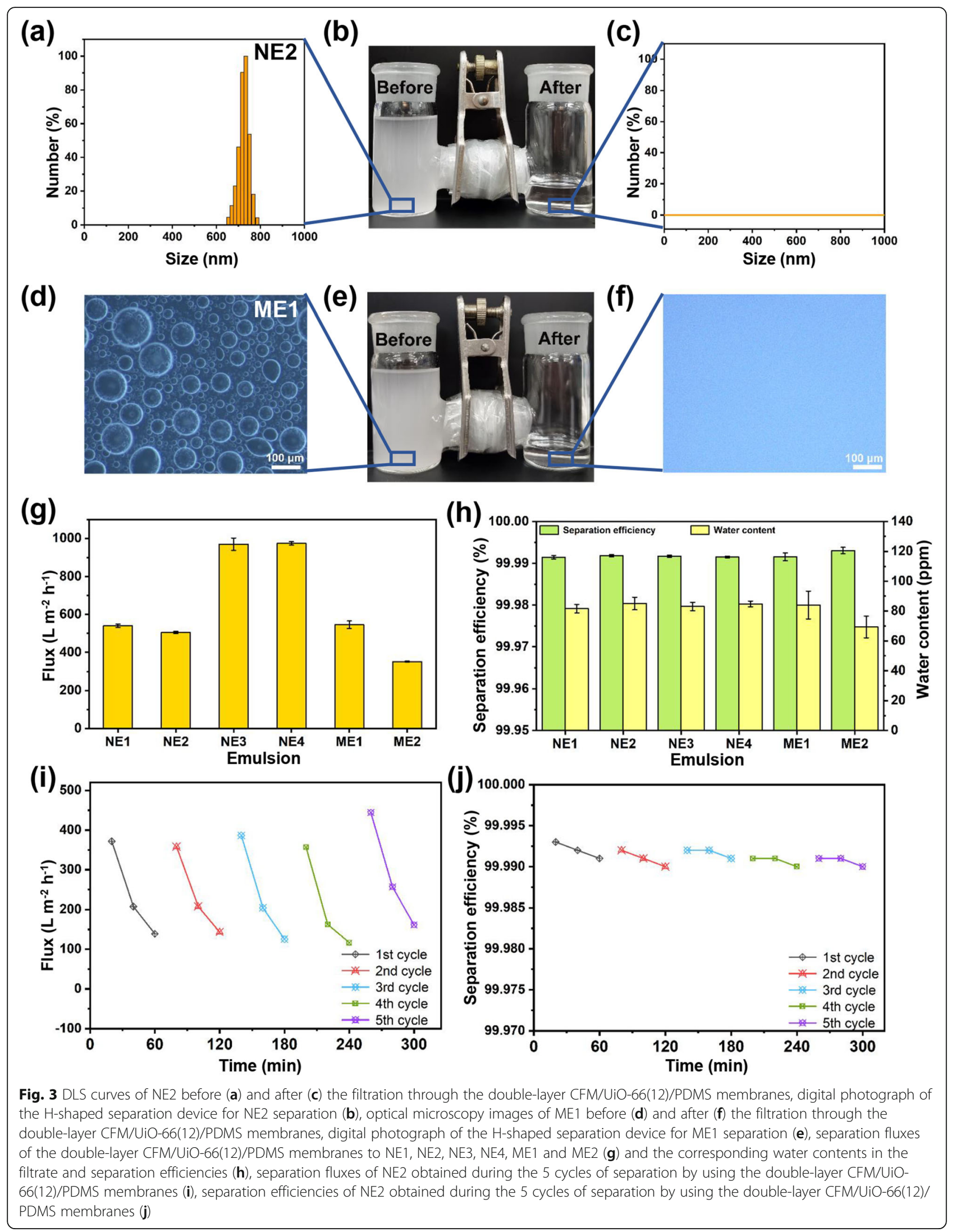


corresponding separation efficiencies of $99.991 \%$, $99.992 \%, 99.992 \%, 99.992$ and $99.993 \%$, respectively (Fig. 3h).

We also compared the separation performance of the double-layer CFM/UiO-66(12)/PDMS membranes to that of commercial polytetrafluoro ethylene (PTFE) membrane. The commercial PTFE membrane is superhydrophobic with a WCA of $156.5^{\circ}$, as shown in Figure S10. When commercial PTFE membrane is applied in NE1 separation, the separation efficiency in the filtrate collected by the commercial PTFE membrane is $99.990 \%$ which is comparable to that of the CFM/UiO66(12)/PDMS membrane (99.992\%). However, the flux obtained by the double-layer CFM/UiO-66(12)/PDMS membranes $\left(540.4 \mathrm{~L} \mathrm{~m}^{-2} \mathrm{~h}^{-1}\right.$ ) is one order of magnitude higher than that of commercial PTFE membrane $(77.6 \mathrm{~L}$ $\mathrm{m}^{-2} \mathrm{~h}^{-1}$ ). For the separation of NE4, the separation efficiency of the filtrate separated by commercial PTFE membrane $(99.991 \%)$ is comparable to that the doublelayer CFM/UiO-66(12)/PDMS membranes (99.992\%). The flux of the double-layer CFM/UiO-66(12)/PDMS membranes for NE4 separation $\left(973.3 \mathrm{~L} \mathrm{~m}^{-2} \mathrm{~h}^{-1}\right)$ is 3.18-fold to that of commercial PTFE membrane $\left(305.6 \mathrm{~L} \mathrm{~m}^{-2} \mathrm{~h}^{-1}\right)$. These results indicate that the separation capability of the double-layer CFM/UiO-66(12)/ PDMS membranes is superior to that of commercial PTFE membrane, which should be mainly ascribed to the capillary effect-enhanced separation flux of CFM.

We evaluated the reusability of the double-layer CFM/ UiO-66(12)/PDMS membranes for separation of NE2. The double-layer CFM/UiO-66(12)/PDMS membranes were applied in the continuous separation of NE2 for $60 \mathrm{~min}$, which were regenerated by immersing in $30 \mathrm{~mL}$ of ethanol for $10 \mathrm{~min}$, and subsequently reused for the next cycle. During the 5 cycles of NE2 separation, the separation fluxes maintain at a relatively high level (116.4-443.4 $\mathrm{L} \mathrm{m}^{-2} \mathrm{~h}^{-1}$ ), and the corresponding separation efficiencies are as high as 99.990-99.993\% (Fig. 3i, j). These results manifest the good reusability of the double-layer CFM/UiO-66(12)/PDMS membranes.

In practical applications, the membranes may confront the harsh environmental conditions, such as organic solvent, ultraviolet irradiation, as well as strong acid and alkali solutions. Therefore, it is essential to evaluate the chemical durability of the CFM/UiO-66(12)/PDMS membrane. The solvent resistance of the CFM/UiO-66(12)/PDMS membranes was investigated by immersing them in dodecane solution, $n$-heptane solution or n-octane solution. As shown in Fig. 4a, the superhydrophobicity of membranes is still maintained after the immersion treatment, with the corresponding WCAs all higher than $150^{\circ}$. The inset in Fig. 4b shows the digital photographs of $30 \mu \mathrm{L}$ of water droplets at $\mathrm{pH} 2-12$ on the surface of the CFM/UiO66(12)/PDMS membrane. The water droplets at $\mathrm{pH} 2-12$ can stably stand on the surface of the CFM/UiO-66(12)/ PDMS membrane. The acid-alkali resistance of the membranes was further explored by immersing them in acid or alkali solution for $24 \mathrm{~h}$, with the corresponding WCAs higher than $150^{\circ}$ (Fig. 4b). Then, the CFM/UiO-66(12)/ PDMS membranes were immersed in the solutions at $\mathrm{pH} 2$ (for $2.0 \mathrm{~h}$ ) and $\mathrm{pH} 12$ (for $2.0 \mathrm{~h}$ ), respectively. The membranes immersed in the solution at $\mathrm{pH} 2$ (or $\mathrm{pH} 12$ ) were further assembled as the double-layer $\mathrm{CFM} / \mathrm{UiO}$ 66(12)/PDMS membranes and applied in NE1 separation. No obvious decrease in flux or separation efficiency was observed when the double-layer membranes were applied in the separation of NE1 (Figure S11). These results indicate the good acid-alkali resistance of the CFM/UiO66(12)/PDMS membrane. Evaluation of UV-aging resistance is also important since a non-negligible decrease of hydrophobicity has been reported after some superhydrophobicity surfaces (e.g. hydrocarbons and fluoroalkyl) are exposed to ultraviolet irradiation [24]. The WCA of the CFM/UiO-66(12)/PDMS membrane remains above $150^{\circ}$ after treated by ultraviolet irradiation for $24 \mathrm{~h}$ (Fig. 4a). The chemical durability of the CFM/UiO-66(12)/PDMS membrane is attributed to the protection of PDMS coating and the high chemical stability of UiO-66. In addition, the mechanical durability of the CFM/UiO-66(12)/PDMS membrane was also evaluated after subjected to 200 cycles of abrasion. The water droplet maintains the spherical shape on the abraded surface of the CFM/UiO-66(12)/ PDMS membrane, showing similar shape to that of water droplet on the intact surface of the CFM/UiO-66(12)/ PDMS membrane (Fig. 4c). During the abrasion process, the WCAs of the CFM/UiO-66(12)/PDMS membranes maintain above $150^{\circ}$ (Fig. 4d), indicating the durable superhydrophobicity. When the double-layer CFM/UiO66(12)/PDMS membranes assembled by two CFM/UiO66(12)/PDMS membranes that were treated by abrasion with sandpaper for 200 cycles were further applied in separating above water-in-oil emulsions (NE1, NE2, NE3, NE4, ME1 and ME2), no obvious loss of fluxes or separation efficiencies were observed (Fig. 4e, f).

\section{Conclusions}

In summary, we have successfully fabricated a new type of chemically and mechanically durable superhydrophobic membrane by modifying hierarchically fibrous structured CFM with UiO-66, followed by the PDMS coating. The CFM substrate played the essential role to provide mechanical strength and simultaneously enhanced the transmission of liquids. The UiO-66 served as the sizesieving sites to strengthen the separation ability of CFM. The double-layer CFM/UiO-66(12)/PDMS membranes efficiently separated various mixed surfactant-stabilized water-in-oil emulsions under gravity. The separation efficiencies were higher than $99.99 \%$, and the separation 

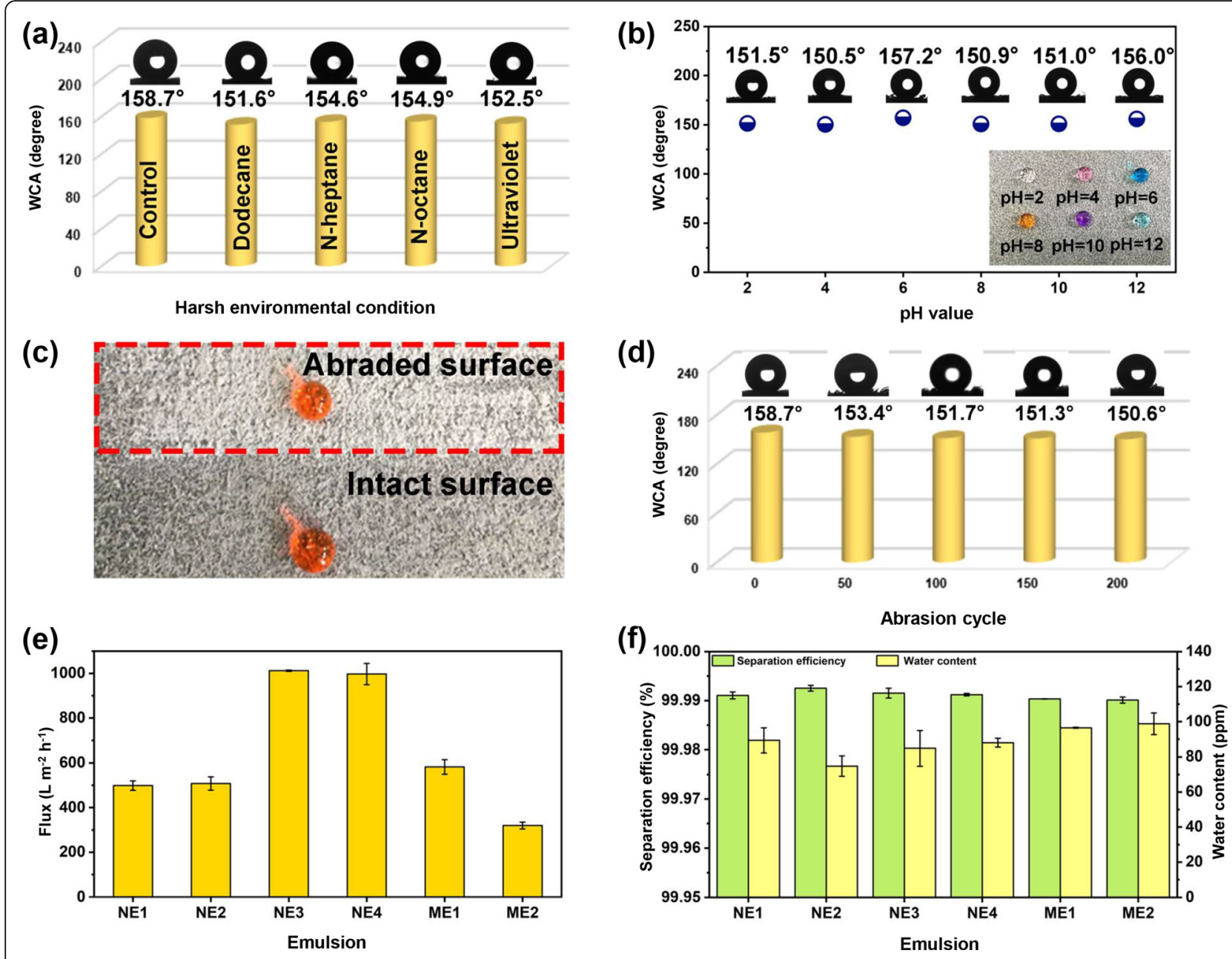

Fig. 4 The WCAs of the CFM/UiO-66(12)/PDMS membrane before and after exposed to different harsh environments for $24 \mathrm{~h}$ (a), the WCAs of the CFM/UiO-66(12)/PDMS membrane immersed in aqueous solutions at varied pH (2-12) for $24 \mathrm{~h}$ (b) (inset in b shows the digital photographs of $30 \mu \mathrm{L}$ of water droplets at $\mathrm{pH} 2-12$ on the surface of the CFM/UiO-66(12)/PDMS membrane), digital photographs of water droplets (30 $\mu \mathrm{L}$ ) on the surface of the CFM/UiO-66(12)/PDMS membrane abraded with sandpaper for 200 cycles (c), the WCAs of the CFM/UiO-66(12)/PDMS membrane abraded with sandpaper for different cycles (d), separation fluxes of the double-layer CFM/UiO-66(12)/PDMS membranes assembled by two membranes that were treated by abrasion with sandpaper for 200 cycles to NE1, NE2, NE3, NE4, ME1 and ME2 (e), the corresponding water contents in the filtrates and the corresponding separation efficiencies(f)

flux reached as high as $973.3 \mathrm{~L} \mathrm{~m}^{-2} \mathrm{~h}^{-1}$, showing evident advantage of separation flux as compared with the commercial polytetrafluoro ethylene membrane. Moreover, the superhydrophobic membrane exhibited good reusability, remarkable chemical stability and mechanical durability. Our research demonstrated a new route for developing high-performance and durable separation membrane system.

\section{Supplementary information}

The online version contains supplementary material available at https://doi. org/10.1186/s42825-021-00060-5.

\section{Additional file 1.}

\section{Acknowledgements}

We thank the financial supports provided by the National Natural Science Funds for Excellent Youth Scholars (No. 21922808), the National Key Research and Development Program (No. 2018YFC1901101), the Fok Ying Tong Education Foundation for Young Teachers in the Higher Education Institutions of China (No. 161099), the National Natural Science Foundation of China (No. 21978176), the 1000 Talents Program of Sichuan Province and the Program of Sichuan University Featured Research Groups in Engineering Disciplines.

\section{Authors' contributions}

Xin Huang and Bi Shi conceived the idea. Huifang Li and Wan Zheng carried out materials preparation. Huifang Li and Hanzhong Xiao carried out emulsion separation. Huifang Li, Baicun Hao and Yujia Wang carried out materials characterization. Huifang Li, Wan Zheng, Xin Huang and Bi Shi drafted the manuscript. All authors analyzed the experimental results. The author(s) read and approved the final manuscript. 


\section{Funding}

National Natural Science Funds for Excellent Youth Scholars (No. 21922808), the National Key Research and Development Program (No. 2018YFC1901101), the Fok Ying Tong Education Foundation for Young Teachers in the Higher Education Institutions of China (No. 161099), the National Natural Science Foundation of China (No. 21978176) and the 1000 Talents Program of Sichuan Province.

\section{Availability of data and materials}

Not applicable.

\section{Declarations}

\section{Competing interests}

The authors declare that they have no known competing financial interests or personal relationships that could have appeared to influence the work reported in this paper.

Received: 2 March 2021 Accepted: 11 May 2021

Published online: 15 June 2021

\section{References}

1. Ashrafizadeh SN, Motaee E, Hoshyargar V. Emulsification of heavy crude oil in water by natural surfactants. J Pet Sci Eng. 2012:86:137-43.

2. Pei L, Wu P, Liu J, Wang J. Effect of nonionic surfactant on the microemulsifying water in silicone media. J Surfact Deterg. 2017;20:247-54.

3. Cheng J, Yu Y, Zhu M. Enhanced biodegradation of sugarcane bagasse by clostridium thermocellum with surfactant addition. Green Chem. 2014;16: 2689-95.

4. Glavac Nk, Lunder M. Preservative efficacy of selected antimicrobials of natural origin in a cosmetic emulsion. Int J Cosmet Sci. 2018;40:276-84.

5. Zhu Y, Wang J, Zhang F, Gao S, Wang A, Fang W. Zwitterionic nanohydrogel grafted PVDF membranes with comprehensive antifouling property and superior cycle stability for oil-in-water emulsion separation. Adv Funct Mater. 2018;28:1804121.

6. Li HJ, Cao YM, Qin JJ, Jie XM, Wang TH, Liu JH, Yuan Q. Development and Characterization of Anti-Fouling Cellulose Hollow Fiber UF Membranes for Oil-Water Separation. J Membr Sci. 2006;279:328-35.

7. Padaki M, Murali RS, Abdullah MS, Misdan N, Moslehyani A, Kassim MA. Membrane technology enhancement in oil-water separation: a review. Desalination. 2015;357:197-207

8. Crick CR, Gibbins JA, Parkin IP. Superhydrophobic polymer-coated coppermesh: membranes for highly efficient oil-water separation. J Mater Chem A. 2013:19:5943-8

9. Gao S, Shi Z, Zhang W, Zhang F, Jin j. Photoinduced superwetting singlewalled carbon Nanotube/ $/ \mathrm{TiO}_{2}$ ultrathin network films for ultrafast separation of oil-in-water emulsions. ACS Nano. 2014;8:6344-52.

10. Gao S, Zhu Y, Zhang F, Jin J. Superwetting polymer-decorated SWCNT composite ultrathin films for ultrafast separation of oil-in-water nanoemulsions. J Mater Chem A. 2015;3:2895-902.

11. Striemer CC, Gaborski TR, McGrath JL, Fauchet PM. Charge- and size-based separation of macromolecules using ultrathin silicon membranes. Nature. 2007:445:749-53.

12. Deng D, Tang R, Liao X, Shi B. Using collagen fiber as a template to synthesize hierarchical mesoporous alumina fiber. Langmuir. 2008;24:36870.

13. Navarro D, Wu J, Lin W, Fullana-i-Palmer P, Puig R. Life cycle assessment and leather production. J Leather Sci Eng. 2020;2:1-13.

14. Han Y, Hu J, Jiang L. Collagen skin, a water-sensitive shape memory material. J Mater Chem B. 2018;6:5144-52.

15. Wells HC, Sizeland KH, Kirby N, Hawley A, Mudie S, Haverkamp RG. Collagen fibril structure and strength in acellular dermal matrix materials of bovine, porcine, and human origin. ACS Biomater Sci Eng. 2015;1:1026-38.

16. Xiao H, Cui Y, Wang Y, Li H, Chen G, Huang X, Shi B. Synergistic combination of the capillary effect of collagen fibers and size-sieving merits of metal-organic frameworks for emulsion separation with high flux. Ind Eng Chem Res. 2020;59:14925-34.

17. Furukawa H, Cordova KE, O'Keeffe M, Yaghi OM. The chemistry and applications of Metal-Organic frameworks. Science. 2013;341:1230444.
18. He J, Zhang Y, Pan Q, Yu J, Ding H, Xu R. Three metal-organic frameworks prepared from mixed solvents of DMF and HAc. Microporous Mesoporous Mat. 2006;90:145-52.

19. James SL. Metal-organic frameworks. Chem Soc Rev. 2003:32:276-88.

20. Liu H, Liu Y, Li Y, Tang Z, Jiang H. Metal-Organic framework supported gold nanoparticles as a highly active heterogeneous catalyst for aerobic oxidation of alcohols. J Phys Chem C. 2010;114:13362-9.

21. DeStefano MR, Islamoglu T, Garibay SJ, Hupp JT. Room-Temperature synthesis of UiO-66 and thermal modulation of densities of defect sites. Chem Mater. 2017:29:1357-61.

22. Cavka JH, Jakobsen S, Olsbye U, Guillou N, Lamberti C, Bordiga S, Lillerud K. $P$. A new zirconium inorganic building brick forming metal organic frameworks with exceptional stability. J Am Chem Soc. 2008;130:13850-1.

23. Chen C, Chen D, Xie S, Quan H, Luo X, Guo L. Adsorption behaviors of organic micropollutants on zirconium metal-organic framework UiO-66: analysis of surface interactions. ACS Appl Mater Interfaces. 2017;9:41043-54.

24. Xiu YH, Hess DW, Wong CP. UV and thermally stable superhydrophobic coatings from Sol-Gel processing. J Colloid Interface Sci. 2008;326:465-70.

\section{Publisher's Note}

Springer Nature remains neutral with regard to jurisdictional claims in published maps and institutional affiliations.

\section{Submit your manuscript to a SpringerOpen ${ }^{\circ}$ journal and benefit from:}

- Convenient online submission

- Rigorous peer review

- Open access: articles freely available online

- High visibility within the field

- Retaining the copyright to your article

Submit your next manuscript at $\boldsymbol{\nabla}$ springeropen.com 